\title{
A Toolkit to Study Sensitivity of the Geant4 Predictions to the Variations of the Physics Model Parameters
}

K.L.Genser, R.Hatcher, G.Perdue, H.Wenzel, J.Yarba (Fermilab)

M.Kelsey, D.H.Wright (SLAC)

On behalf of the Geant4 Collaboration

ACAT 2017, Seattle, WA

$08 / 22 / 2017$ 


\section{General Information (I)}

- Geant4 is the leading detector simulation toolkit used to model interactions between particles and matter, to design detectors and to optimize calibration and reconstruction software in such domains as high energy and nuclear physics, space science, reactor safety, medical applications

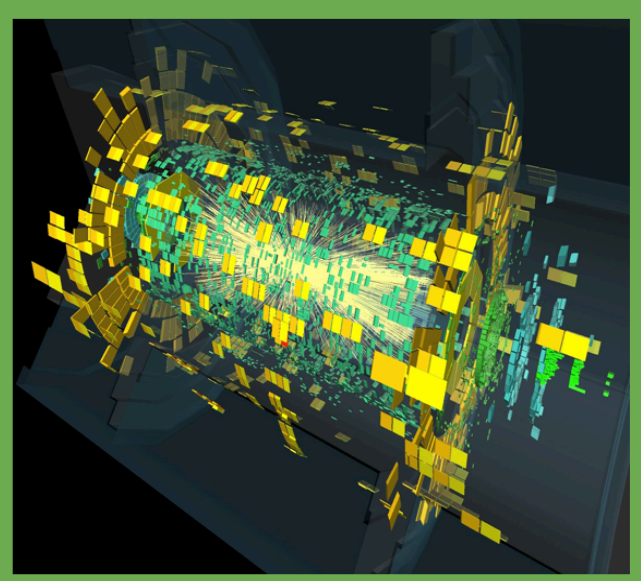

High Energy and Nuclear

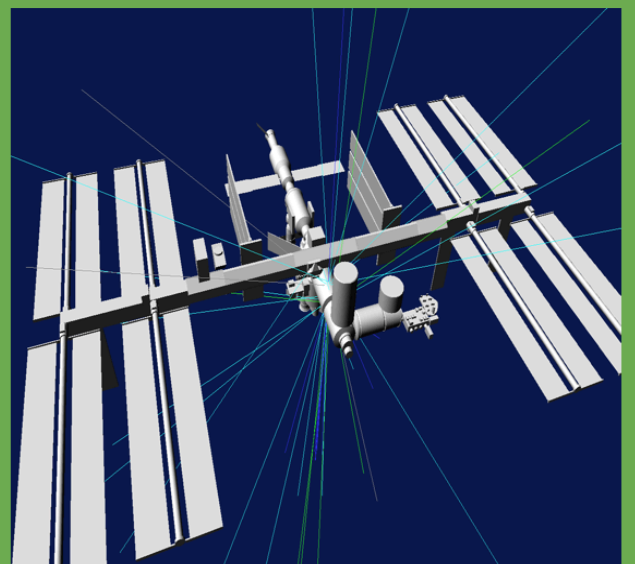

Space Science

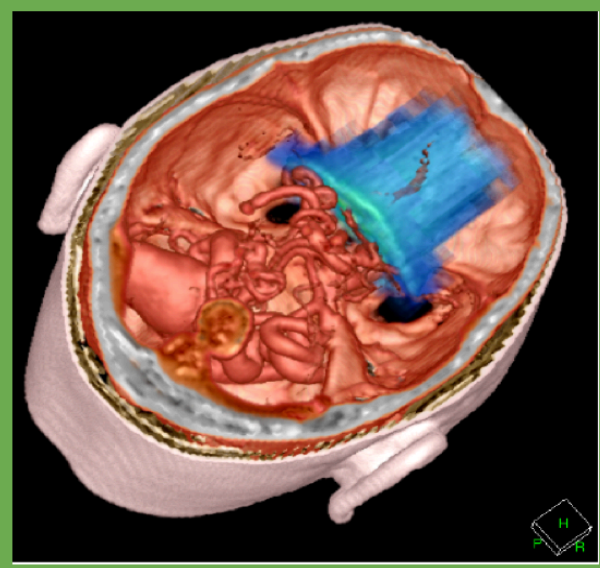

Medicine and Biology 


\section{General Information (II)}

- Geant4 employs a set of validated physics models that span a wide range of interaction energies

\begin{tabular}{|c|}
\hline CHIPS \\
\hline At rest \\
Absorption \\
$\mu, \pi, \mathrm{K}$, anti-p \\
\hline
\end{tabular}

CHIPS (gamma)

$\mu, \pi, \mathrm{K}$, anti-p

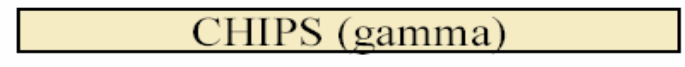

High precision neutron

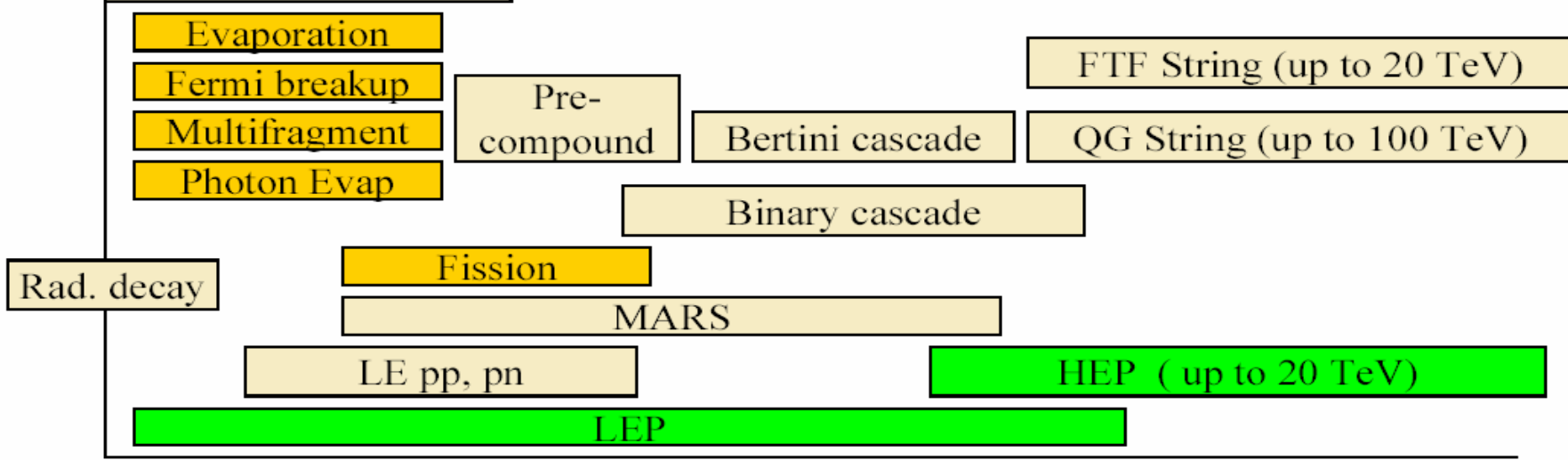

$1 \mathrm{MeV}$

$10 \mathrm{MeV}$

$100 \mathrm{MeV}$

$1 \mathrm{GeV}$

$10 \mathrm{GeV}$

$100 \mathrm{GeV}$

$1 \mathrm{TeV}$ 


\section{General Information (III)}

- Geant4 models rely on directly measured cross-sections and phenomenological models with physically motivated parameters, and are tuned to cover a very wide range of possible experiments

- Because Geant4 can not offer a single modeling algorithm to cover the entire energy domain from zero to the TeV scale, for all known processes and particles, a combination of models is typically used to perform a simulation task

- At present among key Geant4 hadronic models, Fritiof (FTF), Bertini Cascade, and Precompound, there are 39 configurable parameters and 17 switches

- More parameters are likely to be made configurable in the future 


\section{Challenge}

- Critical question: how sensitive are Geant4 predictions to the variations of model parameters, and what uncertainties are associated with a Geant4 physics model, or a group of models, involved in simulation and optimization of a detector design

- Validity ranges of parameters need to be refined

- Correlations of parameters have not been explicitly determined

- The challenge has motivated the Geant4 collaboration to start a new initiative and develop a toolkit to study the effects of varying model parameters on the simulated results, and to explore the associated uncertainties 


\section{Toolkit Features}

- Simulation with multiple configurations of Geant4 model(s) in the multiparameters space

- Collective analysis of multiple variants of the resulting physics observables of interest and simultaneous benchmarking vs relevant experimental datasets (one or many, as applicable)

- Programmatic $(\mathrm{C}++)$ access to the DoSSiER Repository, to extract experimental data for benchmarking and/or statistical analysis:

- http://g4validation.fnal.gov:8080/DoSSiER

- Based on Art Framework: http://art.fnal.gov

- Scientific workflow framework that provides a common software layer to store and access scientific data and to schedule algorithms in an efficient and convenient way 


\section{Software Components and Workflow}

The event processing chain can be executed in one step, or it can be subdivided into multiple steps, if desired

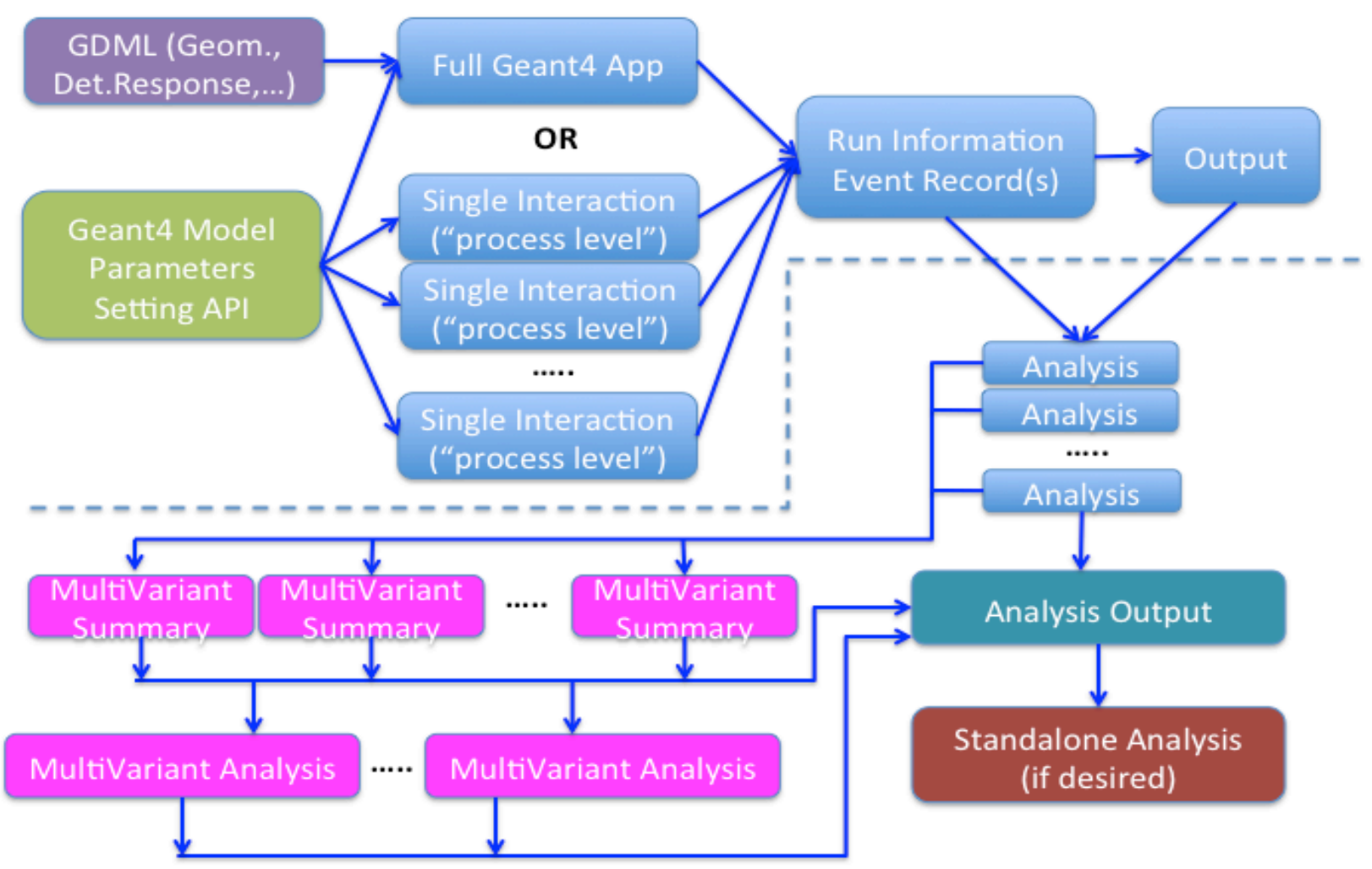




\section{Current Simulation and Analysis}

- Random sampling in 1- or multi-parameter space

- In this study we varied one parameter at a time, 100 settings uniformly distributed with validity range of each parameter

- Simulated events from all "variants" of a Geant4 model can be analyzed "on the fly" or stored for further (re)analysis

- Analysis of various aspects of simulated hadron production in hadronnucleus interactions and benchmarking vs one or multiple experimental datasets

- How much Geant4 predictions vary with one or another parameter

- What settings of model parameters bring MonteCarlo closer to the data

- Can we narrow (at least, qualitatively) validity ranges of parameters

- Results are presented as $\chi^{2}$ as a function of parameter value

- $\chi^{2}=\Sigma\left(\left(X_{i}-Y_{i}\right)^{2} /\left(\sigma_{X}{ }^{2}+\sigma_{Y}{ }^{2}\right)\right), X=$ Data, $Y=$ MonteCarlo, $i=1, N$ where $N$ is the number of experimental data points 


\section{Preliminary Results (I)}

Longitudinal profile of a simulated hadronic shower induced by $2 \mathrm{GeV} / \mathrm{c} \pi^{+}$ incident on Liquid Argon detector volume of the LArIAT setup

$2 \mathrm{GeV} / \mathrm{c} \pi^{+}$on LAr (LArIAT sensitive volume)

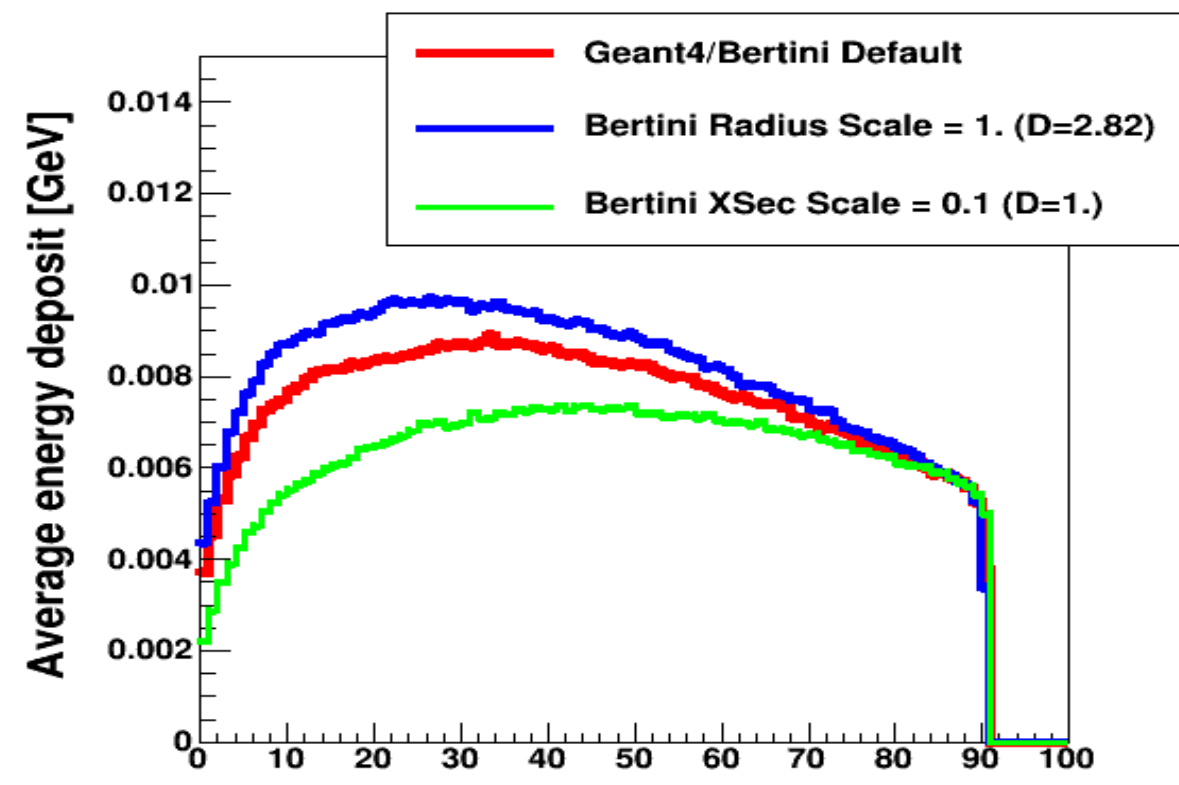

Z-position in the LAr volume [cm]
QGSP_FTFP_BERT physics list is used in the simulation. Bertini Cascade model covers energy range from 0 . to $9.9 \mathrm{GeV}$.

Bertini default predictions are compared with results from variants.

Radius Scale of Bertini is a factor applied to the nuclear radius.

Xsec Scale of Bertini is multiplicative factor applied to the internal cross sections that an incident particle sees as it travels though the target nucleus.

Variations of parameters have been exaggerated to make the effects of changes easier to see. 


\section{Preliminary Results (II)}

\section{Production of proton and $\pi^{+}$by a $5 \mathrm{GeV} / \mathrm{c}$ proton beam interacting with a Lead}

nucleus as simulated by Geant4 Bertini Cascade model and its variants
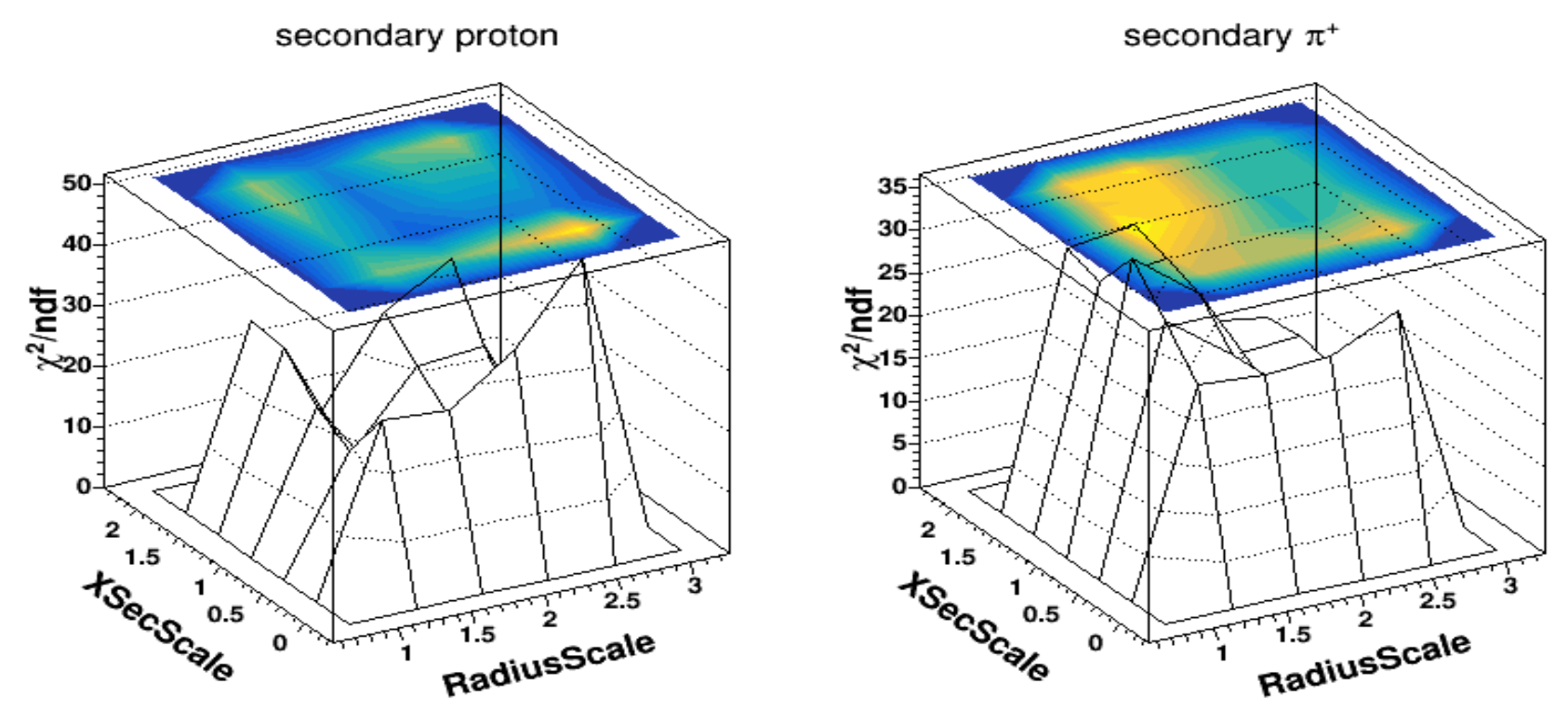

$\chi^{2}$ is calculated for $\mathrm{Ed}^{3} \sigma / \mathrm{dp}^{3}$ (protons) and $\mathrm{d}^{2} \sigma / \mathrm{d} \theta \mathrm{dp}$ (pions) Data: ITEP-83-148 Sov.J.Nucl.Phys., 42, 116 Phys.Rev.C77, 055207, 2008 Phys.Rev.C80, 035208, 2009

Varying multiple parameters may indicate minima in the $\chi^{2}$ distributions that are different from default settings of the parameters and from what a scan by one parameter would favor. 


\section{Preliminary Results (III)}

Production of proton and $\pi^{+}$by a $5 \mathrm{GeV} / \mathrm{c}$ proton beam interacting with a Lead nucleus as simulated by variants of the Geant4 Bertini Cascade model

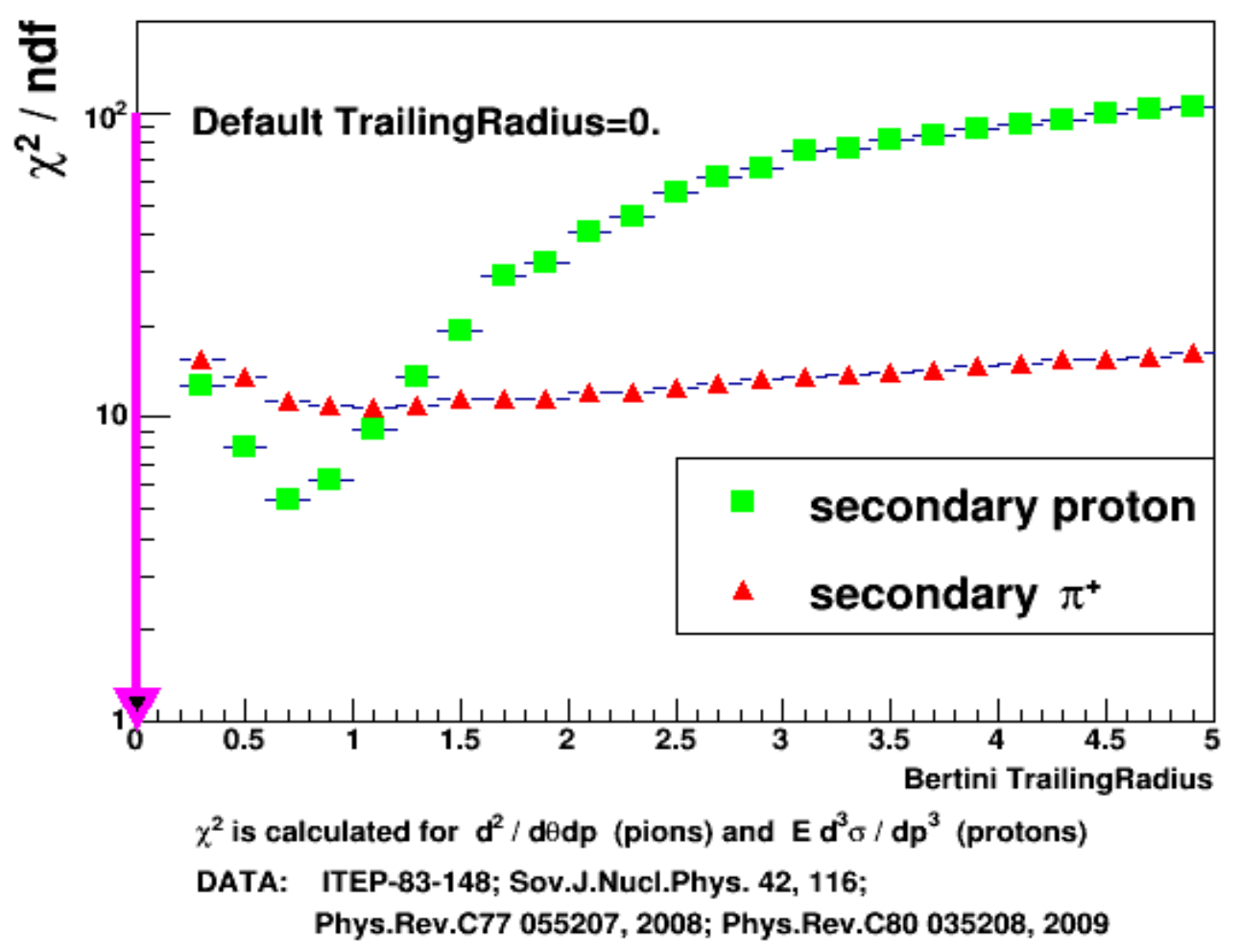

Trailing effect takes into account the local depletion of nuclear density following an intra-nuclear collision.

It is roughly the radius (in $\mathrm{fm}$ ) of the depleted region.

From varying one parameter, it is tempting to claim that a particular value is the best.

Results are obtained with Geant4.10.3.p01 


\section{Preliminary Results (IV)}

Production of proton and $\pi^{+}$by a $5 \mathrm{GeV} / \mathrm{c}$ proton beam interacting with Lead nucleus as simulated by variants of the Geant4 Bertini Cascade model

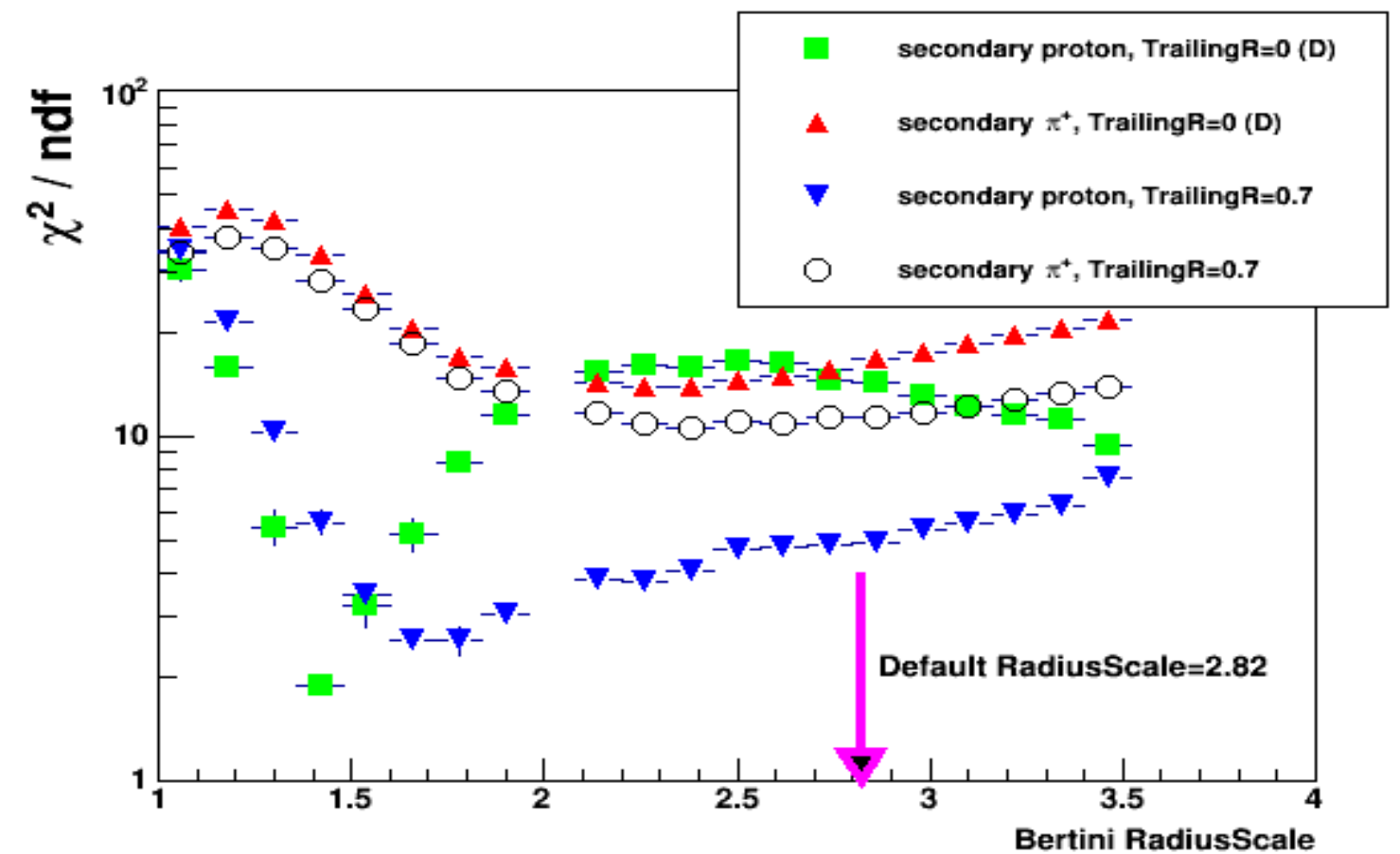

Scans by RadiusScale are done for two different settings of

TrailingRadius.

Results show substantially different behavior of the $\chi^{2}$ distributions that favor different settings of RadiusScale.

Results are obtained with Geant4.10.3.p01

$\chi^{2}$ is calculated for $d^{2} / d \theta d p$ (pions) and $E d^{3} \sigma / d p^{3}$ (protons)

DATA: ITEP-83-148; Sov.J.Nucl.Phys. 42, 116;

Phys.Rev.C77 055207, 2008; Phys.Rev.C80 035208, 2009 


\section{Preliminary Results (V)}

Production of proton and $\pi^{+}$by a $5 \mathrm{GeV} / \mathrm{c}$ proton beam interacting with Carbon or Lead nucleus as simulated by variants of the Geant4 FTF model

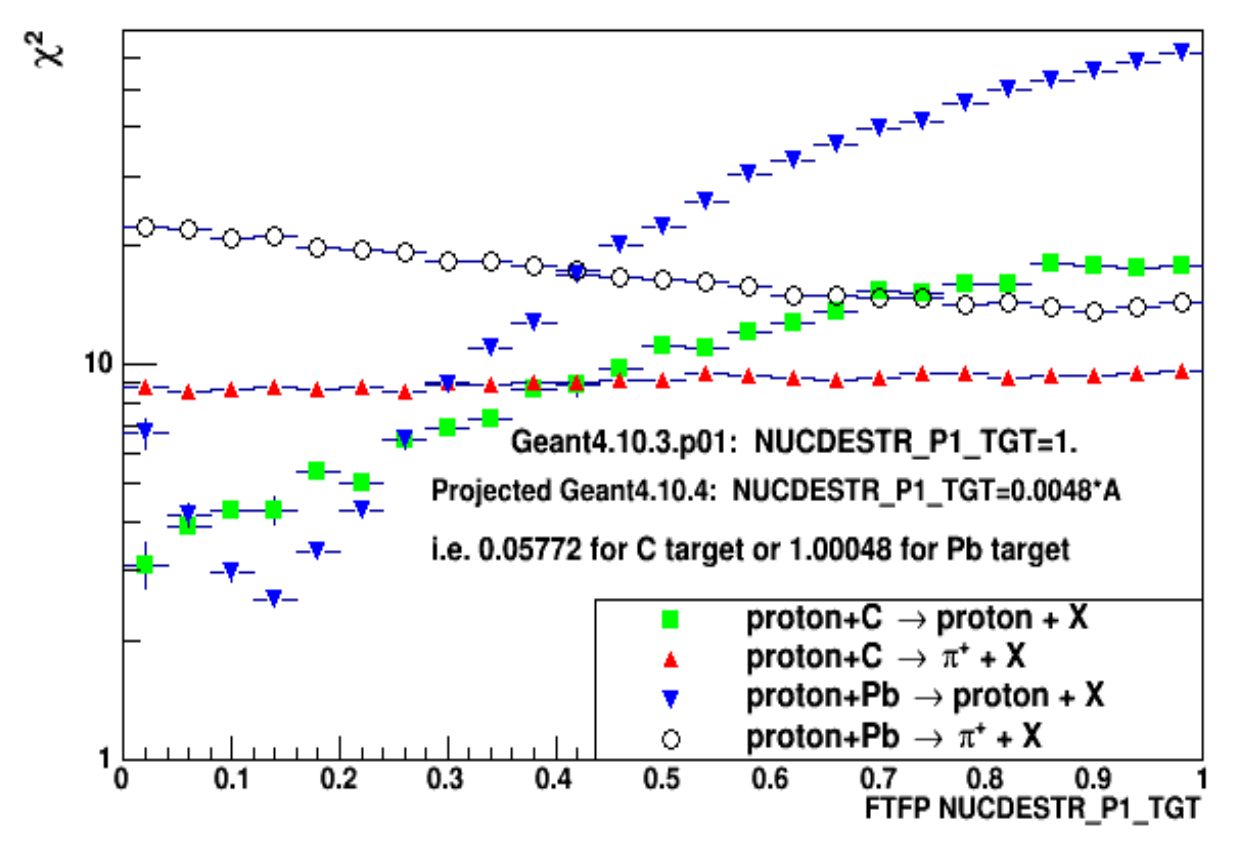

$\chi^{2}$ is calculated for $d^{2} / d \theta d p$ (pions) and $E d^{3} \sigma / d^{3}$ (protons)

DATA: ITEP-83-148; Sov.J.Nucl.Phys. 42, 116;

Phys.Rev.C77 055207, 2008; Phys.Rev.C80 035208, 2009
FTF models nuclear destruction part of hadronnucleus interactions via reggeon cascade. The probability of involving a nuclear nucleon into the cascade

$P\left(\left|s_{i}-s_{j}\right|\right)=C_{n d} \exp \left(-(s i-s j)^{2} / R_{c}^{2}\right)$ $C_{n d}=P 1 \exp (-P 2 *(Y-P 3)) /(1 .+\exp (-P 2 *(Y-P 3)))$ $\mathrm{P} 1$ can be a fixed value (as it is here) or

$\mathrm{P} 1=\mathrm{PO} * \mathrm{~A}$ (target nucleus destruction) $\mathrm{P} 1=\mathrm{PO}$ *BaryonNumber (projectile destruction)

Scan by the same parameter is done for different target nuclei.

Results favor different settings of this parameter which supports the idea of A-dependence.

Results are based on Geant4.10.3.p01 combined with the upcoming updates 


\section{Preliminary Results (VI)}

\section{Production of hadrons by a $158 \mathrm{GeV} / \mathrm{c}$ proton beam interacting with Carbon nucleus as simulated by variants of the Geant4 FTF model}

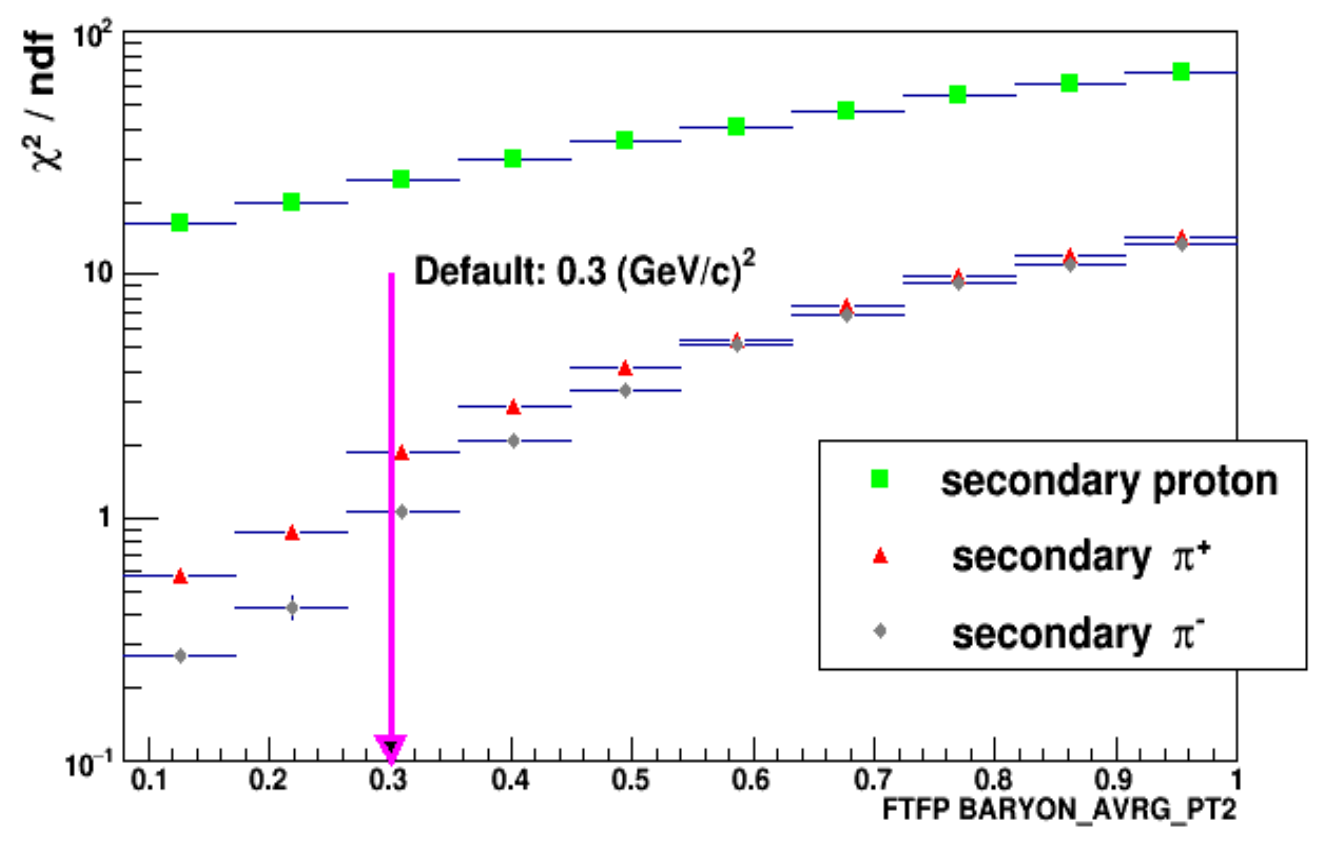

In FTF the excited hadrons are considered as QCD strings that further decays through the fragmentation mechanism.

The $\left\langle\mathrm{p}_{\mathrm{t}}{ }^{2}\right\rangle$ is one of the parameters involved in modeling the excitation process.

A rare case when behavior of the $\chi^{2}$ distributions is similar for all types of secondary particles.

Results are based on Geant4.10.3.p01 combined with the upcoming updates

$\chi^{2}$ is calculated for $\left\langle p_{t}>\right.$ vs $x_{F}$ spectrum

NA49 DATA: https://spshadrons.web.cern.ch/spshadrons 


\section{Current Work and Near-Term Plans}

- The task: to understand errors and correlations of the Geant4 model parameters via simulation and analysis in the multi-parameters space

- Simulation of multiple variants in the multi-parameters space is ready

- The most interesting challenge: analysis in the multi-parameters space, across wide range of energies, projectiles, targets, and secondaries

- Because Geant4 models are tuned to cover a very wide range of possible simulation tasks, they may not always be optimized for a given process or material

- We have interfaced the toolkit to the Professor tuning toolkit:

- http://professor.hepforge.org

- Random sampling in the N-parameter space

- Bin-wise interpolation of MonteCarlo results to $2^{\text {nd }}$ or $3^{\text {rd }}$ order polynomial

- Construction of $c^{2}=S_{\text {bin }}(\text { (interpolation-data })^{2} /$ error $\left.^{2}\right)$ and numerical minimization

- More results are expected shortly for the key Geant4 hadronic models: FTF, Bertini, and PreCompound 


\section{Summary}

- In response to requests from the user community, we are developing a software toolkit to explore the impact of varying Geant4 model parameters on the simulated results

- The toolkit has been used to study effects of varying parameters of the Geant4 Bertini Cascade and FTF models on simulated observables

- The toolkit allows to compare simulated results with experimental data, including statistical analysis

- Experimental data are programmatically obtained from DoSSiER repository

- Selected results and comparison with experimental data have been shown

- Further development include use of the Professor tuning toolkit for analysis in the multi-parameters space

- Last but not least:

- Given the complexity of the models, we do not recommend to use these functionalities to "retune" Geant4 for a better agreement with the data of a particular experiment at this time

- Because this is a developing activity, with some of the functionalities to become available only in the upcoming release, users who are willing to study the model parameters impact are strongly encouraged to contact Geant4 model developers for guidance and feedback 